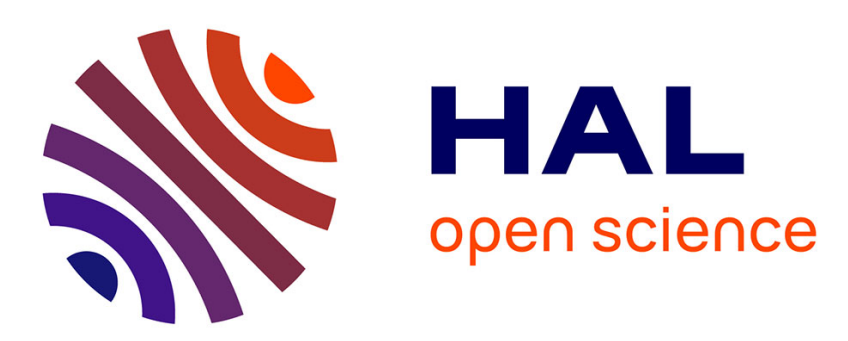

\title{
Altered myofilament structure and function in dogs with Duchenne muscular dystrophy cardiomyopathy
}

Younss Ait Mou, Alain Lacampagne, Thomas Irving, Valérie Scheuermann, Stéphane Blot, Bijan Ghaleh, Pieter de Tombe, Olivier Cazorla

\section{- To cite this version:}

Younss Ait Mou, Alain Lacampagne, Thomas Irving, Valérie Scheuermann, Stéphane Blot, et al.. Altered myofilament structure and function in dogs with Duchenne muscular dystrophy cardiomyopathy. Journal of Molecular and Cellular Cardiology, 2018, 114, pp.345-353. 10.1016/j.yjmcc.2017.12.008 . hal-02376879

\section{HAL Id: hal-02376879 \\ https://hal.umontpellier.fr/hal-02376879}

Submitted on 22 Nov 2019

HAL is a multi-disciplinary open access archive for the deposit and dissemination of scientific research documents, whether they are published or not. The documents may come from teaching and research institutions in France or abroad, or from public or private research centers.
L'archive ouverte pluridisciplinaire HAL, est destinée au dépôt et à la diffusion de documents scientifiques de niveau recherche, publiés ou non, émanant des établissements d'enseignement et de recherche français ou étrangers, des laboratoires publics ou privés. 


\section{Altered myofilament structure and function in dogs with Duchenne muscular dystrophy cardiomyopathy}

Ait Mou et al, Myofilament structure - function in DMD

Younss Ait Mou ${ }^{1}, \mathrm{PhD}$, Alain Lacampagne ${ }^{2}, \mathrm{PhD}$, Thomas Irving ${ }^{1,3}, \mathrm{PhD}$, Valérie Scheuermann ${ }^{2}, \mathrm{MSc}$, Stéphane Blot ${ }^{4}, \mathrm{VMD}, \mathrm{PhD}$, Bijan Ghaleh ${ }^{5}$, PharmD, PhD, Pieter P. de Tombe $1, P h D$, Olivier Cazorla ${ }^{2}, \mathrm{PhD}$

${ }^{1}$ Department of Cell and Molecular Physiology, Heath Science Division, Loyola University Chicago, Maywood, Illinois 60153, USA.

2 INSERM U1046, CNRS UMR9214, Université de Montpellier, PhyMedexp, France.

${ }^{3}$ Department of Biological Sciences, Illinois Institute of Technology, Chicago, Illinois 60616, USA

4 Université Paris-Est, Ecole Nationale Vétérinaire d'Alfort, UPR de Neurobiologie, Maisons-Alfort, France

5 INSERM U 955, Créteil, France

Correspondance to:

Olivier CAZORLA

U1046 INSERM, UMR9214 CNRS, Université de Montpellier

CHU Arnaud de Villeneuve

34295 Montpellier, France

Phone: +33 4674152 44, Fax: +33 4674152 42, olivier.cazorla@inserm.fr 


\begin{abstract}
Aim: Duchenne Muscular Dystrophy (DMD) is associated with progressive depressed left ventricular (LV) function. However, DMD effects on myofilament structure and function are poorly understood. Golden Retriever Muscular Dystrophy (GRMD) is a dog model of DMD recapitulating the human form of DMD.

Objective: The objective of this study is to evaluate myofilament structure and function alterations in GRMD model with spontaneous cardiac failure.

Methods and results: We have employed synchrotron x-rays diffraction to evaluate myofilament lattice spacing at various sarcomere lengths (SL) on permeabilized LV myocardium. We found a negative correlation between SL and lattice spacing in both subepicardium (EPI) and sub-endocardium (ENDO) LV layers in control dog hearts. In the ENDO of GRMD hearts this correlation is steeper due to higher lattice spacing at short SL $(1.9 \mu \mathrm{m})$. Furthermore, cross-bridge cycling indexed by the kinetics of tension redevelopment (ktr) was faster in ENDO GRMD myofilaments at short SL. We measured post-translational modifications of key regulatory contractile proteins. S-glutathionylation of cardiac Myosin Binding Protein-C (cMyBP-C) was unchanged and PKA dependent phosphorylation of the CMyBP-C was significantly reduced in GRMD ENDO tissue and more modestly in EPI tissue.

Conclusions: We found a gradient of contractility in control dogs' myocardium that spreads across the LV wall, negatively correlated with myofilament lattice spacing. Chronic stress induced by dystrophin deficiency leads to heart failure that is tightly associated with regional structural changes indexed by increased myofilament lattice spacing, reduced phosphorylation of regulatory proteins and altered myofilament contractile properties in GRMD dogs.
\end{abstract}

Keywords: Heart Failure; Left Ventricular failure; Length Dependent Activation, Myofilament Properties; Contractile Proteins; Transmural Heterogeneity; Muscular Dystrophy; Hypertrophic Cardiomyopathy 


\section{Introduction}

Duchenne Muscular Dystrophy (DMD) is an X-linked genetic mutation and lethal muscular disorder. This pathology is characterized by loss of dystrophin protein, a protein involved in striated muscle structure and function maintenance ${ }^{1}$. Heart failure constitutes, together with respiratory failure, the main leading cause of mortality in patients with muscular dystrophy ${ }^{2}$. Only a few studies have examined the impact of DMD on cardiac contractility 3,4 . Thus, the cellular molecular mechanisms underlying this myopathy-linked myocardial contractile disorder are poorly understood and yet to be discovered.

In most species, the left ventricle (LV) is characterized by a gradient of contractility across its free wall. The inner LV layer (Endocardium, ENDO) develops higher passive and active contractile properties, when compared to the outer LV layer (Epicardium, EPI) ${ }^{5-8}$. This gradient of contractility is significantly reduced in ischemic heart failure, caused, mainly, by failure in the ENDO layer contractile efficiency ${ }^{8}$. Myofilament contractile heterogeneity is also observed in the heart of healthy Golden Retriever dogs ${ }^{3}$. Similar to rodent models of heart failure, the gradient of transmural contractility is altered in Golden Retriever muscular dystrophy (GRMD) dogs ${ }^{3}$. The GRMD mimics more closely the human disease of DMD compared to other existing mammalian models of dystrophin deficiency ${ }^{9}$. A mutation in the dystrophin gene of GRMD dogs leads to dystrophic muscle lesions, inflammatory foci, progressive fibrosis and fatty infiltration, early locomotor impairment, and premature death due to respiratory or cardiac failure. Our previous report showed that GRMD dogs with heart failure display marked alterations in contractile properties as assessed from myocytes isolated from the ENDO layer. These alterations were correlated with abnormal sarcomeric protein phosphorylation and impaired e/nNOS content ${ }^{3}$. In the present work, we investigated the effect of muscular dystrophy on sarcomere structure and its impact on contractile function. To this aim, we investigated the modulation of cardiac force production by length change, a phenomenon termed lengthdependent activation (LDA) ${ }^{10,11}$, in the GRMD model. LDA forms the cellular basis of the cardiac Frank-Starling mechanism ${ }^{12}$. Increased $\mathrm{Ca}^{2+}$ sensitivity of the sarcomere upon stretch involves complex and dynamic mechanisms at the myofilament level that are still incompletely understood. We found a negative correlation between sarcomere length (SL) and lattice spacing both in EPI and ENDO non-failing wild-type dog myocardium. At short SL, muscular dystrophy induced an increased myofilament lattice spacing and decreased phosphorylation levels of regulatory contractile proteins that are associated with increased myofilament sensitivity and cross-bridge cycling kinetics. We hypothesized that increased myofilament lattice spacing yield to more optimal orientation of myosin heads resulting in improved cross-bridge kinetics.

\section{Methods}

An expanded Methods section is available in the Data Supplement.

\section{Animal model}

The animal care and the experimental protocol were in accordance with the Directive 2010/63/EU of the European Parliament and approved by the local animal ethical committee.

Cardiac tissue was obtained from one-year old GRMD dogs (CEDS, Mézilles, France) with a fractional shortening $\leq 30 \% \quad(n=4)$ and age-matched normal golden retriever dogs 
of the same genetic background $(n=4)$. Conventional echocardiography and 2D color tissue Doppler imaging (TDI) were performed with a Vivid 7 ultrasound unit equipped with 5-7.5 and $2-5 \mathrm{MHz}$ phased-array transducers (GE, Waukesha, WI) as previously described ${ }^{3}$. After sacrifice (pentobarbital, $100 \mathrm{mg} / \mathrm{kg}$ ), cardiac tissues were collected from the sub-endocardium or sub-epicardium, frozen in liquid nitrogen and stored at $-80^{\circ} \mathrm{C}$ until use.

\section{Permeabilized cardiomyocyte mechanics:}

Myofilament $\mathrm{Ca}^{2+}$ sensitivity and cross-bridge cycling kinetics as indexed by the exponential rate of tension redevelopment $\left(\mathrm{k}_{\mathrm{tr}}\right)$ were measured in single permeabilized cardiomyocytes isolated from frozen dog LV myocardium as previously described ${ }^{3,6}$. The Force-calcium relationship was studied_at either 1.9 or $2.3 \mu \mathrm{m}$ sarcomere length $(\mathrm{SL})^{8}$. Kinetic of tension redevelopment $\left(\mathrm{k}_{\mathrm{tr}}\right)$ was measured by mechanically disrupting forcegenerating cross-bridges induced by rapid release/restretch protocol at either submaximal activating solution $\left(\left[\mathrm{Ca}^{2+}\right]=1.3 \mu \mathrm{mol} / \mathrm{L}\right)$ or at maximal calcium activation $\left(\left[\mathrm{Ca}^{2+}\right]=32 \mu \mathrm{mol} / \mathrm{L}\right)^{5}$. For each cardiomyocyte, at a given $\mathrm{SL}$, the above protocol was repeated 3 times and the average $\mathrm{ktr}$ was estimated

\section{Myofilament lattice spacing:}

Permeabilized LV myocardium was prepared according to previous reports ${ }^{13}$. Frozen LV was rapidly thawed, homogenized in ice-cold relaxing solution using Polytron homogenizer (3s, 1,000 RPM; Power Gen 700D; Fisher Scientific), and permeabilized with $1 \%$ Triton $\mathrm{X}-100$ containing relaxing solution overnight at $4^{\circ} \mathrm{C}$.

X-ray diffraction experiments were conducted at the BioCAT undulator-based beamline 18ID at the Advanced Photon Source (Argonne National Labs, Lemont, ${ }^{14}$ ). The permeabilized muscle bundles $(\sim 3-5 \mathrm{~mm})$ were selected based on their shape (i.e. well defined edges) and striation visibility. Lattice spacing was measured as described previously ${ }^{15}$. The sample to detector distance for the small-angle X-ray diffraction camera was $\sim 3 \mathrm{~m}$ and the focal spot size was about $150 \times 50 \mu \mathrm{m}$ in the detector plane. Diffraction images were collected on a high sensitivity CCD-based X-ray detector with $39 \mu \mathrm{m}$ pixels and an active area of $80 \times 160 \mathrm{~mm}$. Exposure times were $\sim 1 \mathrm{~s}$ with an incident X-ray flux of $\sim 1 \times 10^{12}$ photons/s. Lattice spacing $\left(d_{1,0}\right)$ was evaluated from the diffraction pattern using Bragg's law. The ratio of the intensities of the 1,1 and 1,01 equatorial reflections $\left(\mathrm{I}_{11}, \mathrm{I}_{10}\right)$ were measured from nonlinear least square fits to 1-dimensional projections of the integrated intensity along the equator. X-ray reflections were assumed to have a Gaussian peak shape with the widths constrained by those expected for a paracrystalline hexagonal lattice. ${ }^{16,17}$ To evaluate the length effect on myofilament lattice spacing $x$-rays diffraction experiments were conducted on relaxed muscles $(\sim 1.9 \mu \mathrm{m} \mathrm{SL})$ and following stretch to various SL.

Western blot analysis.

Myocardial protein expression was studied, as previously described. ${ }^{18}$ Solubilized proteins in non-reducing Laemmli buffer were separated using SDS-PAGE electrophoresis and were revealed overnight with primary antibodies (see Supplemental Table S1). Bands were revealed and quantified with the Odyssey system (LI-COR Biosciences, Lincoln, Nebraska) 


\section{Results:}

Effect of sarcomere length on lattice spacing in golden retriever dog model:

To evaluate trans-mural contractile heterogeneity in non-failing dog hearts, myocardium samples were isolated from both EPI and ENDO left ventricular layers. Here we tested whether myofilament lattice spacing responds differently to stretch in ENDO and EPI tissues. We obtained clear X-ray patterns from our tissue sample showing sharp equatorial reflections (Figure 1).
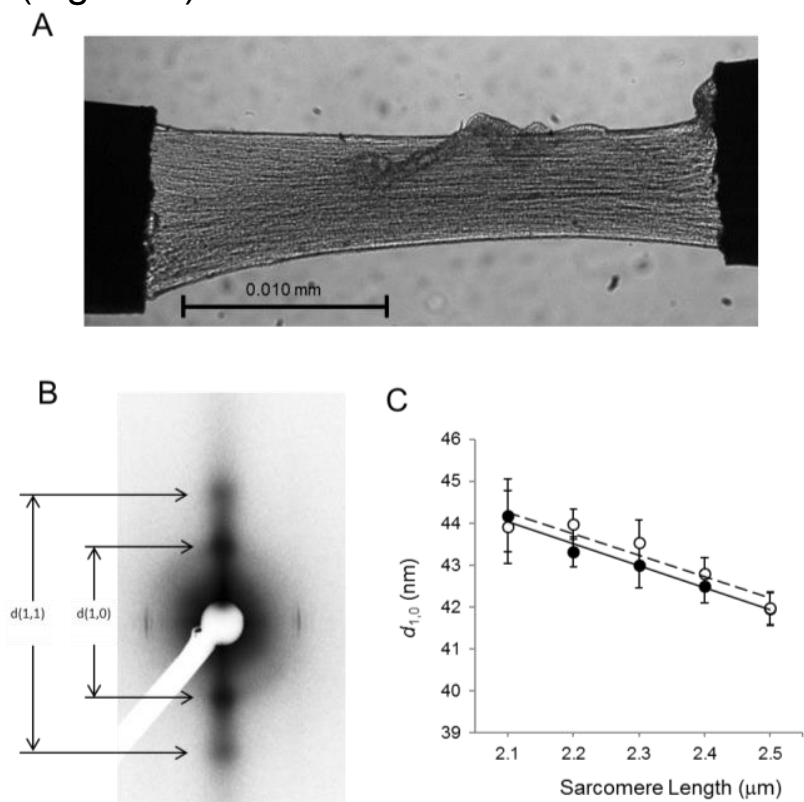

Figure 1: Transmural myofilament lattice spacing in myocardium from a dog animal model. A-B) Typical CCD image showing LV free wall permeabilized muscle strip $(A)$ and equatorial diffraction pattern obtained from an ENDO dog myocardium (B). C) Myofilament lattice spacing $\left(d_{1,0}\right) v s$. SL relationships obtained in ENDO (open circle) and EPI (solid circle) control dog myocardium. (Data areexpressed as mean \pm SEM, $\mathrm{N}=5$ )

As expected, spacing between thin and thick filament was reduced upon stretch. We found that $d_{1,0}$ decreased linearly with $S L$ over the $S L$ range from 2.1 to $2.5 \mu \mathrm{m}$ in ENDO and EPI. The slope of the $S L-d_{1,0}$ relationship was similar in both myocardial tissue layers (Figure 1). To study the correlation between lattice spacing and LDA, we investigated sarcomere function in isolated myocytes from the same hearts by evaluating the myofilament $\mathrm{Ca}^{2+}$ sensitivity at short and long SL. Both ENDO and EPI cardiomyocytes showed a decreased $\mathrm{EC}_{50}$ at long SL indicating increased $\mathrm{Ca}^{2+}$ sensitivity (Figure 2, B). To estimate LDA, we computed the difference between $\mathrm{EC}_{50}$ at short $(1.9 \mu \mathrm{m})$ and long $(2.3 \mu \mathrm{m}) \mathrm{SL}\left(\mathrm{EC}_{50}\right)$. This parameter is commonly employed to evaluate LDA. We found LDA to be higher in ENDO cardiomyocytes (Figure 2, C) as indexed by a significantly higher $\triangle \mathrm{EC}_{50}$ in this region. These results indicate that in healthy dog myocardium, the higher length sensitivity of activation in the inner layer of the ventricle cannot be explained by a differential interfilament lattice spacing. 


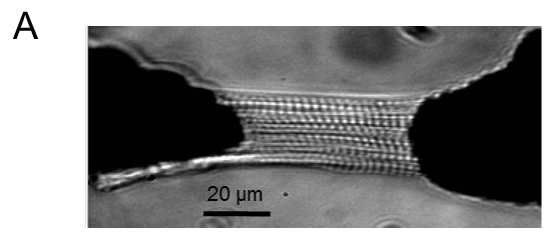

B

C
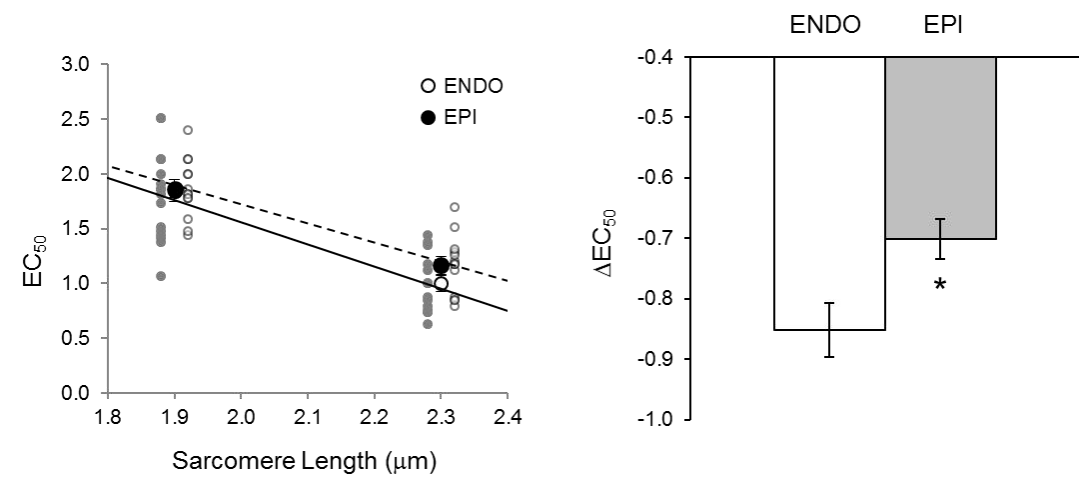

Figure 2: Transmural Length Dependent Activation (LDA) in permeabilized cardiomyocyte isolated from a dog heart. A) Typical CCD image showing a permeabilized dog myocyte. B) A typical negative relationship between $\left[\mathrm{Ca}^{2+}\right]$ generating half of maximal active force $\left(E_{50}\right)$ and $S L(1.9$ and $2.3 \square \mathrm{m})$ on EPI (open circle) and ENDO (solid circle) cardiomyocytes. Regression lines were obtained from the average of individual regression lines. C) LDA is evaluated by computing $\square \mathrm{EC50}=\mathrm{EC}_{50}$ (2.3 um SL)-EC50 (1.9um SL).

( $\mathrm{N}=4$ cells per animal, 4 animals per group). ${ }^{*} v s$. ENDO; $\mathrm{P}<0.05$.

\section{Effect of DMD on cardiac myofilament $\mathrm{Ca}^{2+}$ sensitivity and lattice spacing:}

To evaluate the severity of heart failure in GRMD, echocardiography measurement of LV function was performed. Our result showed significant reduction of the Fractional Shortening (FS, Table 1).

\begin{tabular}{lll}
\hline & Control & GRMD \\
\hline Age (year) & $1.1 \pm 0.1$ & $1.0 \pm 0.0$ \\
Weight $(\mathrm{kg})$ & $26.8 \pm 2.5$ & $16.8 \pm 0.6^{*}$ \\
Heart rate (bpm) & $101 \pm 12$ & $160 \pm 21^{*}$ \\
Fractional Shortening (FS) (\%) & $34.3 \pm 1.7$ & $24.7 \pm 0.9^{*}$ \\
LVDD (mm) & $44.6 \pm 2.5$ & $43.2 \pm 3.7$ \\
LVDD/BW & $1.7 \pm 0.1$ & $2.6 \pm 0.2^{*}$ \\
$\mathrm{~N}$ & 4 & 4 \\
\hline
\end{tabular}

Table 1: Echocardiographic data obtained from Control (non-failing) and GRMD (failing) dogs. Left Ventricular End Diastolic Diameter (LVDD); Body Weight (BW). Data are represented as mean \pm SEM (* vs. Control; $p<0.05)$.

We evaluated myofilament $\mathrm{Ca}^{2+}$ sensitivity and LDA on ENDO permeabilized cardiomyocytes isolated from both non-failing control dogs and GRMD with cardiac failure. Myofilament $\mathrm{Ca}^{2+}$ sensitivity was higher at short SL in ENDO GRMD myocytes compared with control dogs as indexed by a significant lower EC50 (Figure 3A). 
Differences of myofilament $\mathrm{Ca}^{2+}$ sensitivity between non-failing and GRMD ENDO myocytes disappeared following stretch. As a result, LDA indexed by the average $\mathrm{EC}_{50}$ was lower in GRMD myocytes (Figure 3B). We did not find any significant difference in EPI cardiomyocytes contractile parameters (data not shown) as we previously reported ${ }^{19}$. To determine whether the changes in myofilament $\mathrm{Ca}^{2+}$ sensitivity in ENDO myocytes from failing hearts were associated with myofilament structure alteration, we performed small-angle X-ray diffraction experiments on permeabilized control dog and GRMD endocardial myocardium at short $(\sim 1.9 \mu \mathrm{m})$ and long $(\sim 2.3 \mu \mathrm{m})$ sarcomere length ${ }^{13,15}$. Figure $3 \mathrm{C}$ shows a typical CCD captured image of $X$-ray diffraction pattern. Pixel intensity was plotted and the 1,0 equatorial reflection derived lattice spacing estimated (Figure 3D). As observed with the first experiments (Figure 1), the lattice spacing reduces with stretch in both CTRL and GRMD myocardium (Figure 3D). Interestingly, at short SL, the interfilament lattice spacing was significantly higher in GRMD ENDO myocardium $(46.22 \pm 0.31 \mathrm{~nm})$ compared to control non-failing myocardium $(43.81 \pm 0.26 \mathrm{~nm})$. Stretch eliminates this difference by inducing an ENDO GRMD interfilament lattice spacing reduction to a value that matches the spacing obtained in control non-failing dog myocardium (42.91 $\pm 0.20 \mathrm{~nm}$ in CTRL vs. $42.90 \pm 0.46 \mathrm{~nm}$ in GRMD).

A

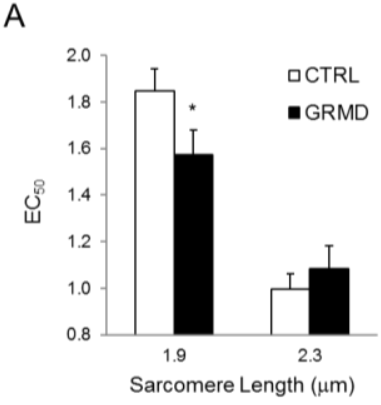

C
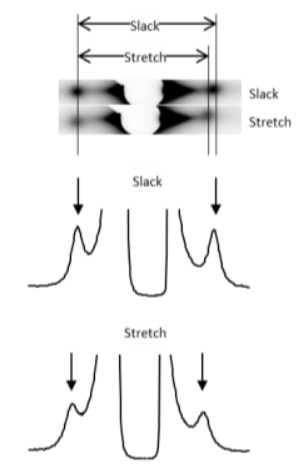

B

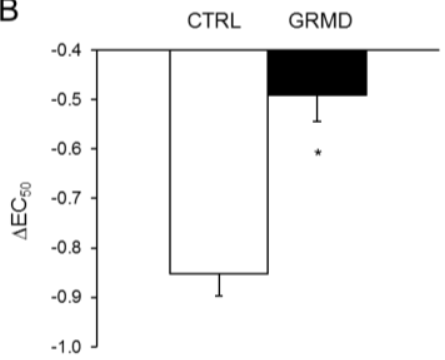

D

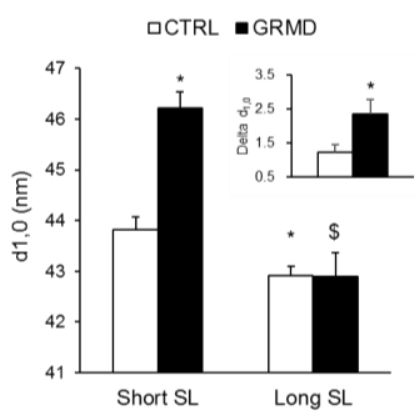

Figure 3: Effect of myopathy on myofilament properties of ENDO permeabilized cardiomyocytes. Myofilament $\mathrm{Ca}^{2+}$ sensitivity was indexed by $\mathrm{EC}_{50}(\mathrm{~A})$ and LDA (B) were evaluated on CTRL (open bars) and GRMD (solid bars) ENDO permeabilized cardiomyocytes at short $(1.9 \mu \mathrm{m})$ and long $(2.3 \mu \mathrm{m}) \mathrm{SL}$. $(\mathrm{N}=4$ cells per animal, 4 animals per group). * vs. CTRL; $P<0.05$. C) Representative $X$-ray and intensity profile of permeabilized GRMD myocardium as recorded at slack length and after stretch (+20\% $\left.L_{0}\right)$. D) Average myofilament lattice spacing as measured on ENDO myocardium of CTRL (open bars) and GRMD (solid bars) at slack length (Short SL) and after 20\%Lo stretch (Long SL). (N=4 animals per group). * vs. CTRL short SL; $\$$ vs. GRMD short SL; $P<0.05$. 
The myopathy induced a myofilament lattice expansion at short length that exceeded the normal physiological range. In order to test if the interfilament spacing expansion was not due to the lack of dystrophin we also analyzed the myocardium dissected from the subepicardium. We did not find any significant difference between GRMD and CTRL EPI myocardium at both short SL $(43.61 \pm 0.48 \mathrm{~nm}$ vs. $44.55 \pm 0.91 \mathrm{~nm}$, respectively) and long SL $(42.47 \pm 0.36 \mathrm{~nm}$ vs. $43.28 \pm 0.93 \mathrm{~nm}$, respectively). Therefore, we conclude that the higher lattice spacing in ENDO GRMD myocardium may be involved in the increased myofilament $\mathrm{Ca}^{2+}$ sensitivity observed at short SL independently of dystrophin absence. Equatorial intensity ratios $\left(l_{11}, l_{10}\right)$, under relaxed conditions were not significantly different under all conditions studies indicating that the relative degree of association of myosin heads with actin under relaxed conditions were similar in all samples. To further study the effect of lattice spacing on the myofilament contractile performance, we evaluated the cross-bridge cycling kinetics.

\section{Myopathy in GRMD alters cross-bridge properties}

We measured the kinetics of tension redevelopment $(\mathrm{ktr})$ in both non-failing and GRMD permeabilized ENDO cardiomyocytes (Figure 4). $K_{t r}$ is usually used to estimate the rate of transition from weakly bound (non-force-generating) to strongly bound (forcegenerating) cross-bridges. Therefore, an estimation of cross-bridge cycling performance can be obtained. In ENDO cardiomyocytes from healthy dogs, $k_{\text {tr }}$ obtained at sub-maximal $\mathrm{Ca}^{2+}$ activation tended to increase after stretch but did not reach significance (Figure 4 B). Interestingly, cross-bridge cycling redevelopment was accelerated in ENDO GRMD myocytes only at short SL as indicated by the higher $\mathrm{ktr}_{\mathrm{tr}}$ (Figure 4B). No significant effect between groups was found on cross-bridges kinetics at maximal calcium activation, nor in EPI myocardium under all conditions (Figure 4B). Collectively, our results suggest that the structural changes that occurred in myocytes from GRMD dog with heart failure were associated with altered myofilament contractile properties. 
A

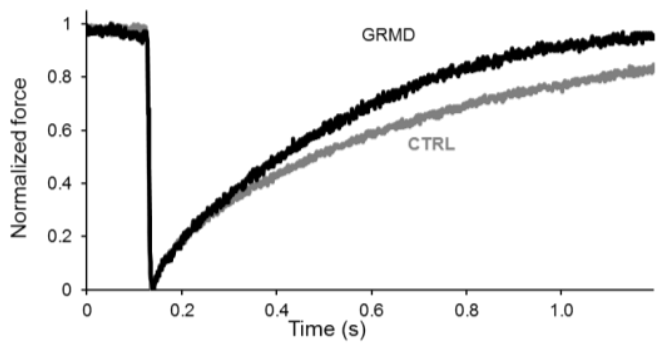

B
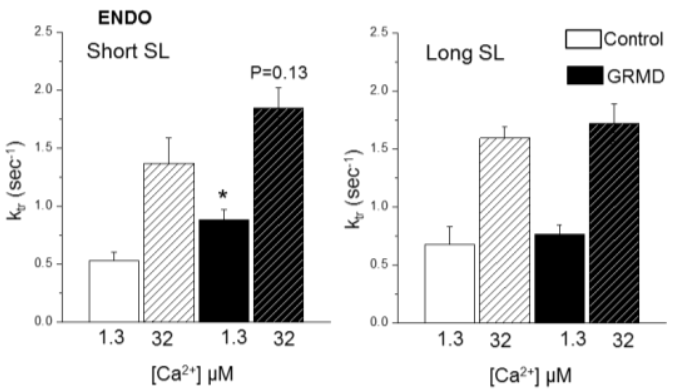

C
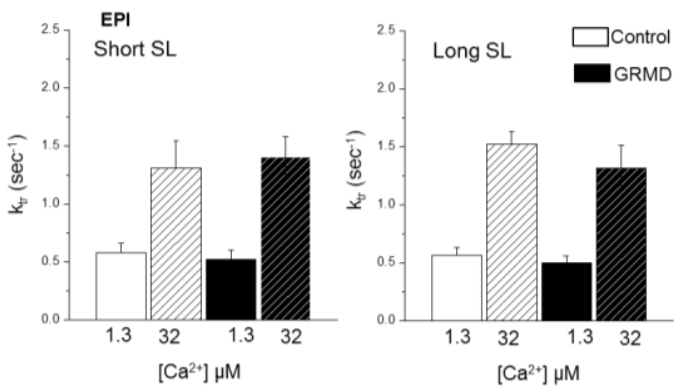

Figure 4: Effect of myopathy on the rate of tension redevelopment $\left(\mathrm{k}_{\mathrm{tr}}\right)$. A) Raw tension recording obtained following rapid (2ms) $20 \%$ initial muscle length shortening of CTRL (gray trace) and GRMD (black trace) permeabilized cardiomyocyte. B-C) Average $\mathrm{K}_{\text {tr }}$ obtained from CTRL (white bars) and GRMD (gray bars) ENDO (B) and EPI (C) tissue at short $(1.9 \mu \mathrm{m})$ and long $(2.3 \mu \mathrm{m}) \mathrm{SL}$ at maximal [Ca $\left.{ }^{2+}\right]$ (hashed bars) and submaximal [ $\left.\mathrm{Ca}^{2+}\right]$ (solid bars). ( $\mathrm{N}=4$ cells per animal, 4 animals per group) ${ }^{*} v s$. CTRL; $P<0.05$.

\section{Intracellular signaling pathways:}

To further understand the intracellular mechanisms involved in the GRMD myocytes contractile dysfunction, we measured post-translational modifications of key regulatory contractile proteins. We measured the PKA dependent phosphorylation of the cardiac Myosin Binding Protein-C (cMyBP-C) at Ser-284, which was significantly reduced in GRMD ENDO tissue and more modestly in EPI tissue (Figure 5A). 
A

ENDOCARDIUM
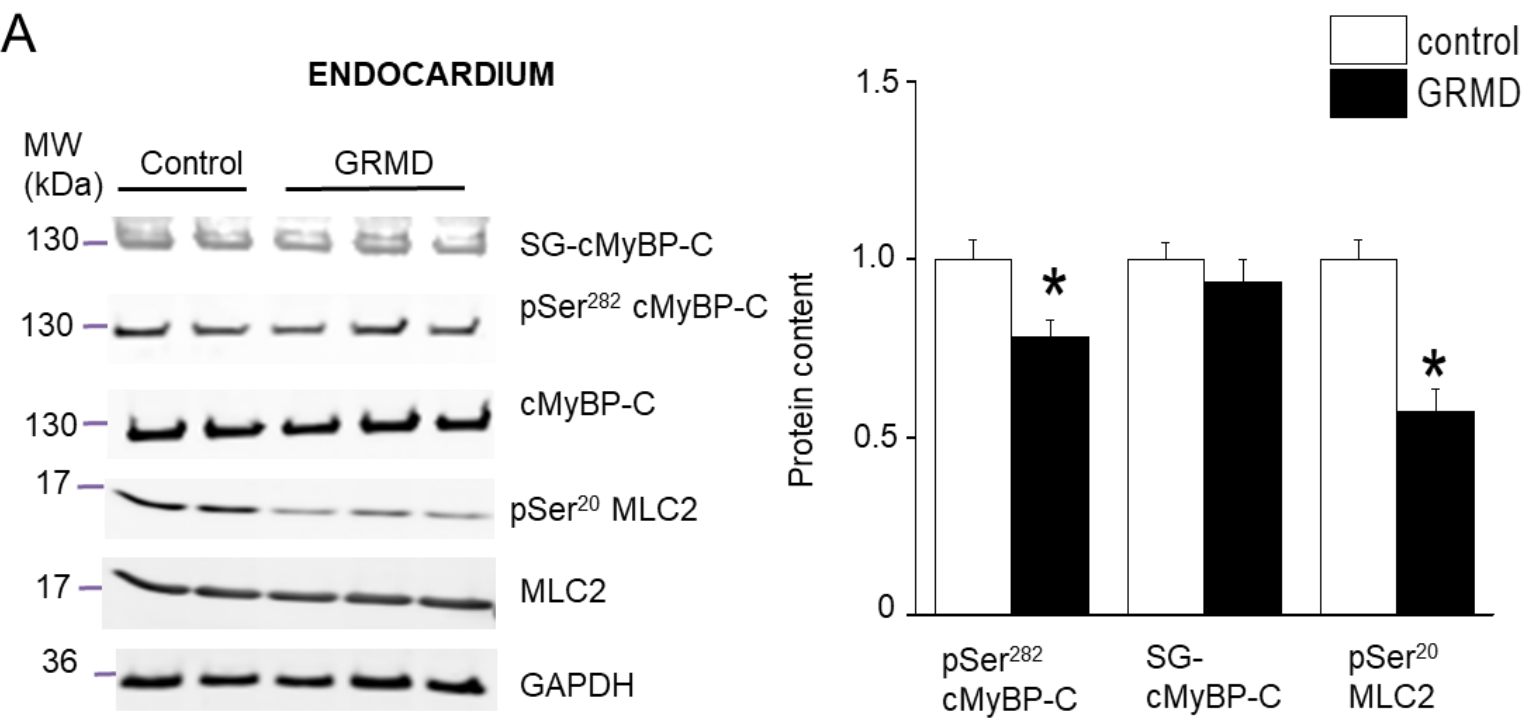

B

EPICARDIUM
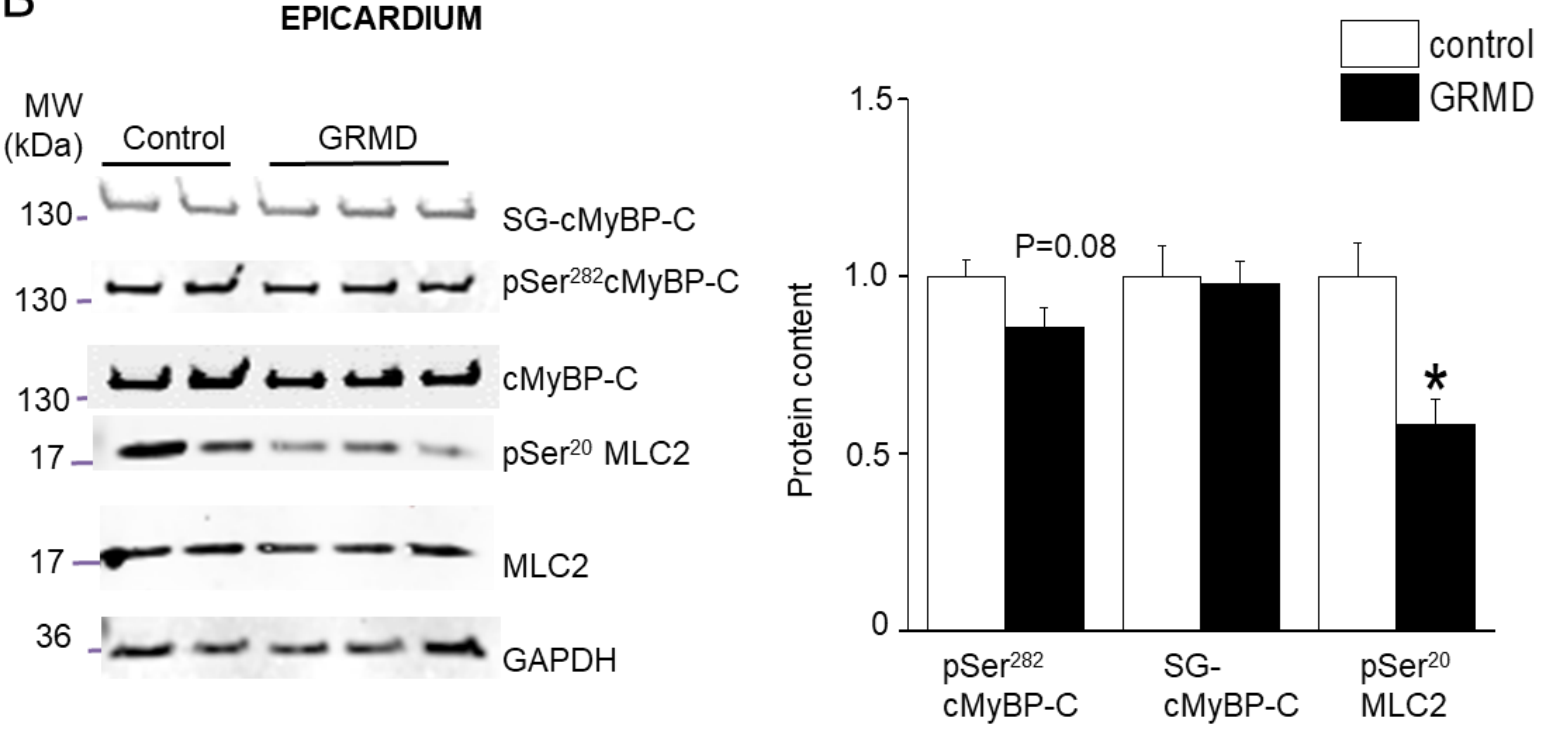

Figure 5: Effect of GRMD on thick filament post-transcription (phosphorylation and glutathionylation) modification. All data are shown for control (open bars) and GRMD (closed bars) ENDO (A) and EPI (B) myocardium. Typical Western blots (left panel) and averaged results (right panel) for cardiac Myosin Binding Protein-C (cMyBP-C) Ser-282 phosphorylation site, cMBP-C Sglutathionylation, and Myosin Light Chain 2 (MLC2) phosphorylation at Ser20 site. All posttranscriptionnal modifications were normalized by the total protein content on the same band. $(\mathrm{N}=4$ animals per group, in duplicate) ${ }^{*}$ vs. CTRL; $\mathrm{P}<0.05$.

We also measured the S-glutathionylation of CMyBP-C that has been shown in vitro to be activated in conditions of oxidative stress and to lead to increased myofilament $\mathrm{Ca}^{2+}$ sensitivity ${ }^{20}$. The level of S-glutathionylated CMyBP-C was similar in both regions of control and GRMD hearts (Figure 5). Next, we studied the myosin light chain 2 (MLC2) whose phosphorylation increases myofilament $\mathrm{Ca}^{2+}$ sensitivity. The pSer20-MLC2 level decreased in both EPI and ENDO GRMD tissues to a similar extent (Figure 5). Troponin 
I phosphorylation is an important modulatory process of cardiac myofilament properties 21. Tnl phosphorylation at the Ser22/23 site decreased significantly in GRMD hearts and to a higher extent in ENDO tissues compared with EPI (Figure 6). The Tnl phosphorylation level at Thr143 site was not altered. Finally, the levels of the dephosphorylated form of cTnl were similar between regions and conditions. Altogether, the results indicate a profound remodeling of the post-translational modifications of all major regulatory contractile proteins investigated in GRMD hearts. CTnl and cMyBP-C exhibited regional alterations that may be responsible of the regional change in myofibrillar structure/function and the resulting lower LDA.

A

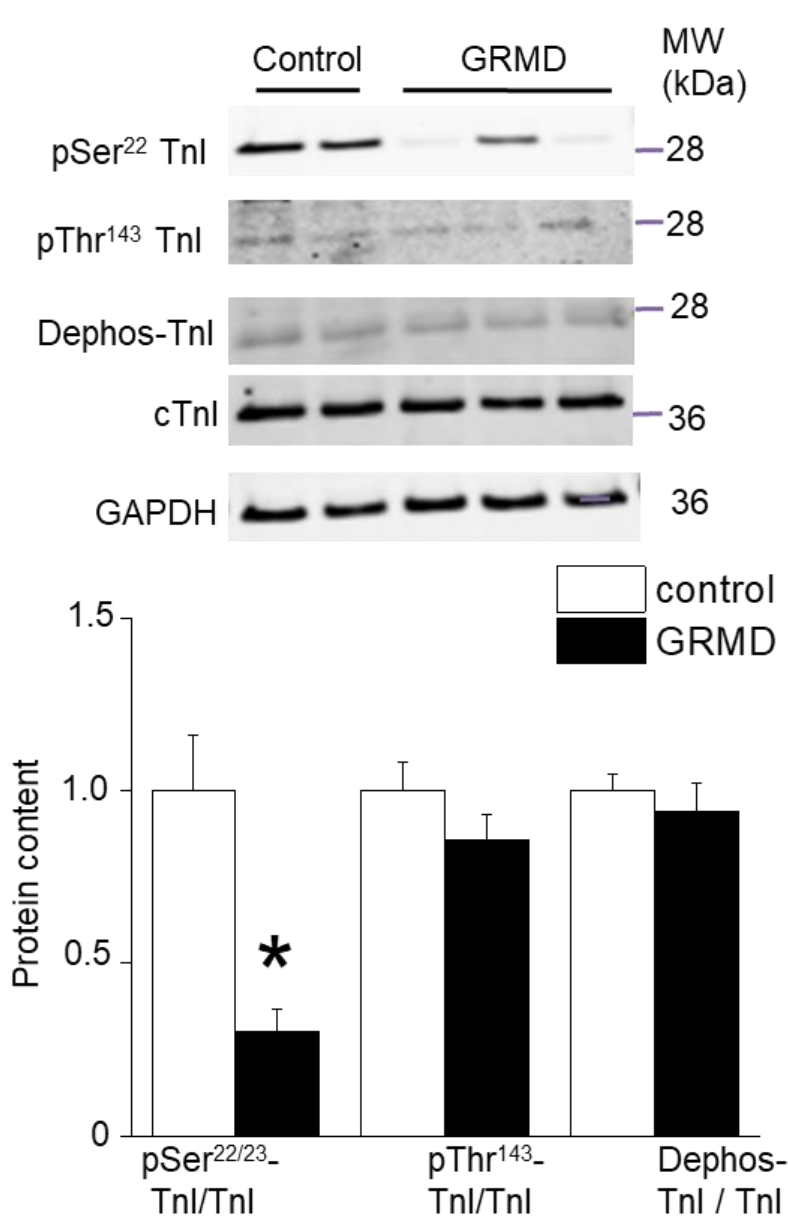

B
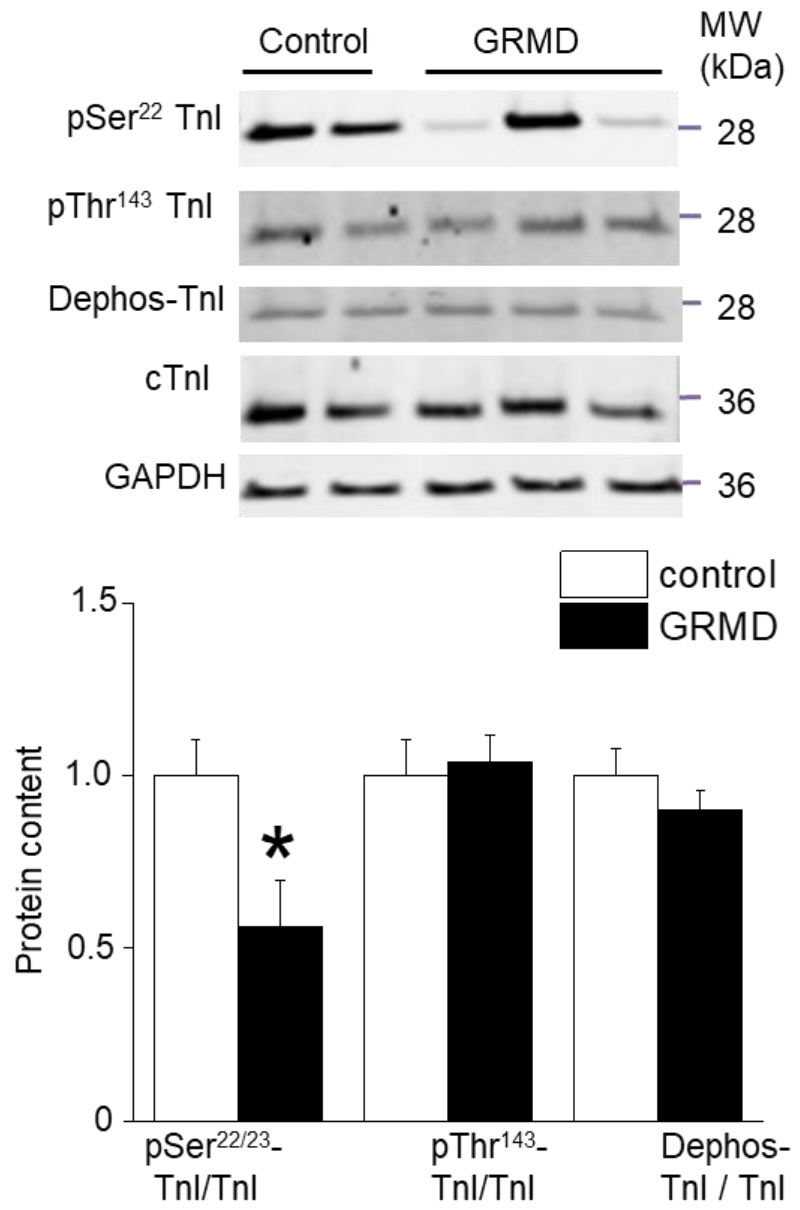

Figure 6: Effect of GRMD on the cardiac Troponin I (cTnl) phosphorylation and dephosphorylation status. All data are shown for control (open bars) and GRMD (closed bars) Endocardium (A) and Epicardium (B) myocardium. The top panels show a typical Western blot for phosphorylation at Ser22/23 and Thr143 and for de-phosphorylation (Dephos) status of the cTnl that have been normalized but the total cTnl content on the same band. The bottom panels show the respective averaged results. ( $\mathrm{N}=4$ animals per group, in duplicate) ${ }^{*}$ vs. $\mathrm{CTRL} ; \mathrm{P}<0.05$. 


\section{Discussion:}

Here, we report the first observations on the contractile machinery structure and function of myocardium isolated from non-failing (CON) and spontaneous muscular dystrophy associated cardiomyopathy Golden Retriever (GRMD) dogs. In normal dog myocardium, the higher length sensitivity of activation observed in the inner layer of the ventricle cannot be explained by different interfilament lattice spacing. Chronic stress induced by the lack of dystrophin leads to heart failure that is tightly associated with regional structural changes, post-translational modifications of regulatory proteins and altered myofilament contractile properties in GRMD dogs.

Synchrotron X-rays diffraction has previously been employed to study myofilament structure-function relationships in different striated muscle types isolated from various species ${ }^{22}$. With stretch, sarcomere length clearly increases while interfilament lattice spacing decreases ${ }^{13,22-24}$. The effect of lattice spacing on LDA of cardiac myofilament has been assessed ${ }^{24-26}$. However, it is still not clear whether the lateral spacing between thin and thick filament directly regulates myofilament contractile performance, particularly following stretch. The reduction of lattice spacing has been first proposed to underlie LDA in the myocardium over the physiological ranges of $S L{ }^{15}$. This hypothesis has been refined a few years later by the same group showing that myofilament lattice spacing alone was not sufficient to explain all stretch-induced alterations of $\mathrm{Ca}^{2+}$ sensitivity in permeabilized myocardium ${ }^{25}$. Most of these studies have been performed in rodents or insects. Rare are studies that investigated cardiac sarcomere structure-function in a larger animal model such as those with bovine hearts ${ }^{24}$. Our present work is the first, to our knowledge, to evaluate the cardiac sarcomere structure-function both in healthy dogs and in dogs with heart failure. Here, we evaluated whether lattice spacing is involved in LDA and cross-bridge cycling kinetic alterations observed in dystrophic dog myocardium. In dogs, we found that lattice spacing decreases with stretch (i.e. increases in SL), which is consistent with the results obtained on other species ${ }^{13,22-24}$. Because of the large amount of tissue available, it was possible in the present work to study, for the first time, regional difference of structure-function across the LV wall. Our present work shows that in the canine animal model, this relationship is identical between the ENDO and EPI region across the LV free wall over the predefined SL range. Moreover, consistent with findings in rodent animal models ${ }^{11}$, we found LDA to be significantly higher in the ENDO myocardium. Thus, under normal conditions, lattice spacing per se cannot explain the stretch-induced regional contractile heterogeneity. Several studies suggested that stretch and LDA could involve other structural changes than interfilament spacing. For instance, changes in myosin head orientation or position relative to the thin filaments can alter LDA without affecting interfilament lattice spacing 27,28 . Our measurements of the equatorial intensity ratio, $11,1 / 11,0$, showed no significant differences in all tissues studied, excluding differences in relative degree of myosin heads with actin as an explanation of our findings. LDA is certainly a complex phenomenon which regulation is not exclusively limited to environment dependency such as post-translational status of the contractile proteins, or titin-based passive tension that have been shown to mediate some structural changes with stretch ${ }^{24,26}$ on the tested species. Moreover, we showed recently that LDA involves titin-based simultaneous structural rearrangements within both thin and thick filaments and not myofilament lattice spacing per $\mathrm{se}^{29}$. In the present study, passive tension is lower in GRMD myocytes $\left(5.1 \pm 0.3 \mathrm{mN} / \mathrm{mm}^{2}\right)$ compared with control myocytes $(7.7 \pm 0.7$ 
$\mathrm{mN} / \mathrm{mm}^{2}$ ). It is thus possible that the reduced LDA observed here involves a titin-based modulation of activation.

With heart failure, we observed at short SL several regional changes in interfilament lattice spacing, myofilament $\mathrm{Ca}^{2+}$ sensitivity and cross-bridge cycling kinetics. These changes might be interconnected. The preferential sub-endocardium alteration, observed here, has been described in several types of heart failure (i.e. Ischemic, Hypertrophic, Congenital) and different animal models (rodent, dog, pig, guinea-pig) 6, 30,31. Several investigations have demonstrated in a normal heart and during disease, regional heterogeneity of the myocardial phenotype and function, including myocardial contractility and the electrophysiological properties of cardiomyocytes. During HF, regional changes in protein expression were reported such as higher atrial natriuretic peptide levels in basal endocardium in a canine model of LV hypertrophy ${ }^{32}$ or lower SERCA2a and PLB transmural expression gradients in the LV free wall endocardium of failing human hearts 33, 34. A transmural gradient of oxidative mitochondrial activity was reported being lower in sub-endocardium associated with higher $\mathrm{NO}$ and ROS productions ${ }^{35}$. Additionally, cardiac hypertrophy ${ }^{35}$, ischemic heart failure ${ }^{18}$, aging and streptozotocin-induced diabetes ${ }^{36}$ might be associated with a decreased transmural gradient in mitochondrial respiratory activity. Several reports from our laboratory and others indicate that the contractile machinery itself can be altered in other types of heart failure indicating that the sarcomere phenotype is not specific to DMD model. It is to note however, that the pattern of alteration differs between models. For instance, in the rodent animal model of ischemic heart failure, the gradient of contractility is altered because of a reduced $\mathrm{Ca}^{2+}$ sensitivity at long sarcomere length in the ENDO layer. Instead, in the present study, we observed that the lost gradient of contractility is mainly due to increased $\mathrm{Ca}^{2+}$ sensitivity a short sarcomere length in the ENDO layer. Nevertheless, both models show the ENDO layer to be specifically affected by various types of heart failure.

There is still debate on what should be the alteration of myofilament $\mathrm{Ca}^{2+}$ sensitivity during heart failure due to contradictory results published with human samples and animal models (see ${ }^{37,} 38$ for debates). Discrepancies may in part result from the duration of the studies (induction of cardiac disease in the course of weeks), compared to long-term studies in our studies (7-8 months duration). It is still discussed that heart failure may be associated with early increase in myofilament $\mathrm{Ca}^{2+}$ sensitivity that reverts to decreased $\mathrm{Ca}^{2+}$ sensitivity at end-stage heart failure, but this needs further study. Mutations in troponin and tropomyosin that cause familial DCM show a consistently lower $\mathrm{Ca}^{2+}$ sensitivity and this was proposed to account for inadequate contractility in familial DCM $\left(\right.$ see for review ${ }^{37}$ ). Decreased myofilament $\mathrm{Ca}^{2+}$ sensitivity in patients suffering from type2 diabetes mellitus compared to patients without diabetes has also been reported 39 . There have been reports of increased myofilament $\mathrm{Ca}^{2+}$ sensitivity in animal models of cardiac disease, both large animal (dog, pig), and small rodents (mouse) ${ }^{38}$. Increased myofilament $\mathrm{Ca}^{2+}$ sensitivity and reduced phosphorylation levels of regulatory proteins is a common feature reported in human heart failure in particular with patients treated with beta-blockers at a stage where $\beta$-adrenergic signaling is defective in failing heart with $\beta$ receptor desensitisation, blunted inotropic response and reduced cardiac reserve. Van der Velden et al showed, using human heart samples with different degrees of failure, a correlation between myofilament $\mathrm{Ca}^{2+}$ sensitivity and regulatory proteins phosphorylation 
such as troponin I ${ }^{40}$ and MLC-2 ${ }^{41}$. A previous report showed in old hypertensive dogs by bilateral renal wrapping a model of HF with preserved ejection fraction, increased myofilament $\mathrm{Ca}^{2+}$ sensitivity and reduced phosphorylation levels of MyBP-C, Tnl and MLC-2 42, similar changes that observed in our study. Thus it appears that hypophosphorylation of sarcomeric proteins could be a general property in the transition to many HF pathologies but not all.

Considering that phosphorylation of either cardiac Tnl or MyBP-C has a large and opposite impact on interfilament function ${ }^{43}$, it is expected that they participate in the regional change of the myofilament structure and function observed in the GRMD dogs. Similar increases in myofilament $\mathrm{Ca}^{2+}$ sensitivity only at short SL have been reported in models of cMyBP-C truncation ${ }^{44-46}$ that was also associated with increased $\mathrm{ktr}_{\mathrm{tr}}{ }^{47}$. Because ktr encompasses both the rates of cross-bridge attachment (f) and detachment (g) ${ }^{48}$, it is impossible from our experiments to determine which step is affected in GRMD dogs. The observed increase in $\mathrm{Ca}^{2+}$ sensitivity at short $\mathrm{SL}$ is likely arising from an increased number of cross-bridges interacting with the thin-filament 47 or may be due to an increase in the apparent $\mathrm{Ca}^{2+}$ binding affinity of $\mathrm{TnC}$ mediated by strongly-bound cross-bridges ${ }^{49-51}$. The increased cross-bridge cycling kinetics observed in the present work may lead to increased myofilament $\mathrm{Ca}^{2+}$ sensitivity through a positive cooperative activation of strongly bound myosin heads on thin filament ${ }^{52}$. Moreover, $\mathrm{k}_{\mathrm{tr}}$ acceleration observed in GRMD ENDO tissues could result from reduced contractile protein phosphorylation. This effect could be accentuated by the observed lattice spacing expansion. Similar results have been observed on insect permeabilized muscle fibers where cross-bridge attachment and detachment kinetics were faster with larger thick-tothin interfilament lattice spacing ${ }^{53}$. Hypophosphorylation of MLC2 is also expected to affect thin filament structure and $\mathrm{Ca}^{2+}$ sensitization ${ }^{54}$. Interestingly, the $\mathrm{D} 166 \mathrm{~V}$ mutation in the MLC2 induces hypertrophic cardiomyopathy with reduced MLC2 phosphorylation, increased myofilament sensitivity ${ }^{55}$ and larger lattice spacing ${ }^{56}$.

From a therapeutic perspective, Taking into account regional alterations of cardiac contractility is certainly an important aspect for future treatment. The new ultrasound technique, speckle-tracking echocardiography (STE) assesses regional myocardial deformations. This technique has clearly shown benefits to detect early contractile defects compared with conventional echocardiography. Early measurement of longitudinal strain by STE revealed in a pig model of acute pressure overload systolic abnormalities upon mild aorta banding despite normal conventional indices of systolic function such as LV fractional shortening and LV dP/dtmax ${ }^{57}$. Endocardial to epicardial velocity gradients measured by tissue Doppler imaging can also be used to identify early LV dysfunction before the ejection fraction is altered. Our results are in agreement with previous report showing that young GRMD dogs exhibited an important decrease in both systolic and diastolic endocardial velocities, whereas epicardial velocities and fractional shortening were not affected ${ }^{58}$. Similarly, myocardial velocity gradient was also lost due to altered endocardial velocities and strains in other models of cardiac pathologies such as ischemic ${ }^{6}$, aortic valve stenosis ${ }^{30,31}$ and hypertensive after abdominal aortic banding, 45 often earlier before heart failure is detected. Treatments will have to target at least the altered layer with minimum impact on the preserved layers. Previous studies indicate that the 
altered layer can be preferentially restored by treatments such as moderate exercise training ${ }^{6}$ or pharmacological calcium sensitizer ${ }^{59}$ and bradykinin ${ }^{3}$. Although interesting, the molecular mechanism underlying this ENDO-specificity is yet to be unraveled.

\section{Conclusion:}

Our study showed transmural differences in sarcomere structure and function in dog myocardium with heart failure, mostly in the lower physiological length range. The increase in myofilament $\mathrm{Ca}^{2+}$ sensitivity observed in GRMD dogs may contribute to the impairment of relaxation during the early phase of diastole observed in this model. Altogether, our findings suggest that impaired LDA may contribute to depressed contractile function in human patients harboring in dystrophin and Duchenne Muscular Dystrophy.

\section{Sources of Funding}

This research used resources of the Advanced Photon Source, a U.S. Department of Energy (DOE) Office of Science User Facility operated for the DOE Office of Science by Argonne National Laboratory under Contract No. DE-AC02-06CH11357. "This project was supported by grant 9 P41 GM103622 from the National Institute of General Medical Sciences of the National Institutes of Health. The content is solely the responsibility of the authors and does not necessarily reflect the official views of the National Institute of General Medical Sciences or the National Institutes of Health. This study was supported by the Association Française contre les Myopathies (AFM) (OC №11590 and AL $\mathrm{N}^{\circ} 15083$ ) and by the $\mathrm{NIH}$ (HL62426).

Disclosures: None 


\section{References}

1. Hoffman EP, Brown RH, Jr. and Kunkel LM. Dystrophin: the protein product of the Duchenne muscular dystrophy locus. Cell. 1987;51:919-28.

2. Finsterer J and Stollberger C. The heart in human dystrophinopathies. Cardiology. 2003;99:1-19.

3. Su JB, Cazorla O, Blot S, Blanchard-Gutton N, Ait Mou Y, Barthelemy I, Sambin L, Sampedrano CC, Gouni V, Unterfinger Y, Aguilar P, Thibaud JL, Bize A, Pouchelon JL, Dabire H, Ghaleh B, Berdeaux A, Chetboul V, Lacampagne A and Hittinger L. Bradykinin restores left ventricular function, sarcomeric protein phosphorylation, and e/nNOS levels in dogs with Duchenne muscular dystrophy cardiomyopathy. Cardiovasc Res. 2012;95:86-96.

4. Townsend D, Turner I, Yasuda S, Martindale J, Davis J, Shillingford M, Kornegay JN and Metzger JM. Chronic administration of membrane sealant prevents severe cardiac injury and ventricular dilatation in dystrophic dogs. J Clin Invest. 2010;120:1140-50.

5. Ait Mou Y, le Guennec JY, Mosca E, de Tombe PP and Cazorla O. Differential contribution of cardiac sarcomeric proteins in the myofibrillar force response to stretch. Pflugers Arch. 2008;457:25-36.

6. Ait Mou Y, Reboul C, Andre L, Lacampagne A and Cazorla O. Late exercise training improves nonuniformity of transmural myocardial function in rats with ischaemic heart failure. Cardiovasc Res. 2009;81:555-64.

7. Cazorla O, Le Guennec JY and White E. Length-tension relationships of sub-epicardial and subendocardial single ventricular myocytes from rat and ferret hearts. J Mol Cell Cardiol. 2000;32:735-44.

8. Cazorla O, Szilagyi S, Le Guennec JY, Vassort G and Lacampagne A. Transmural stretchdependent regulation of contractile properties in rat heart and its alteration after myocardial infarction. FASEB J. 2005;19:88-90.

9. Cooper BJ, Winand NJ, Stedman H, Valentine BA, Hoffman EP, Kunkel LM, Scott MO, Fischbeck $\mathrm{KH}$, Kornegay JN, Avery RJ and et al. The homologue of the Duchenne locus is defective in X-linked muscular dystrophy of dogs. Nature. 1988;334:154-6.

10. Allen DG and Kentish JC. The cellular basis of the length-tension relation in cardiac muscle. $J \mathrm{Mol}$ Cell Cardiol. 1985;17:821-40.

11. Cazorla $\mathrm{O}$ and Lacampagne $\mathrm{A}$. Regional variation in myofilament length-dependent activation. Pflugers Arch. 2011;462:15-28.

12. de Tombe PP, Mateja RD, Tachampa K, Ait Mou Y, Farman GP and Irving TC. Myofilament length dependent activation. J Mol Cell Cardiol. 2010;48:851-8.

13. Witayavanitkul N, Ait Mou Y, Kuster DW, Khairallah RJ, Sarkey J, Govindan S, Chen X, Ge Y, Rajan S, Wieczorek DF, Irving T, Westfall MV, de Tombe PP and Sadayappan S. Myocardial infarction-induced $\mathrm{N}$-terminal fragment of cardiac myosin-binding protein $\mathrm{C}$ (cMyBP-C) impairs myofilament function in human myocardium. J Biol Chem. 2014;289:8818-27.

14. Fischetti R, Stepanov S, Rosenbaum G, Barrea R, Black E, Gore D, Heurich R, Kondrashkina E, Kropf AJ, Wang S, Zhang K, Irving TC and Bunker GB. The BioCAT undulator beamline 18ID: a facility for biological non-crystalline diffraction and X-ray absorption spectroscopy at the Advanced Photon Source. J Synchrotron Radiat. 2004;11:399-405.

15. Irving TC, Konhilas J, Perry D, Fischetti R and de Tombe PP. Myofilament lattice spacing as a function of sarcomere length in isolated rat myocardium. Am J Physiol Heart Circ Physiol. 2000;279:H2568-73.

16. Yu LC, Steven AC, Naylor GR, Gamble RC and Podolsky RJ. Distribution of mass in relaxed frog skeletal muscle and its redistribution upon activation. Biophys J. 1985;47:311-21.

17. Irving TC and Millman BM. Changes in thick filament structure during compression of the filament lattice in relaxed frog sartorius muscle. J Muscle Res Cell Motil. 1989;10:385-94. 
18. Andre L, Fauconnier J, Reboul C, Feillet-Coudray C, Meschin P, Farah C, Fouret G, Richard S, Lacampagne $A$ and Cazorla $O$. Subendocardial increase in reactive oxygen species production affects regional contractile function in ischemic heart failure. Antioxid Redox Signal. 2013;18:1009-20.

19. !!! INVALID CITATION !!! 3.

20. Patel BG, Wilder T and Solaro RJ. Novel control of cardiac myofilament response to calcium by Sglutathionylation at specific sites of myosin binding protein C. Front Physiol. 2013;4:336.

21. Solaro RJ, Henze M and Kobayashi T. Integration of troponin I phosphorylation with cardiac regulatory networks. Circ Res. 2013;112:355-66.

22. Konhilas JP, Irving TC and de Tombe PP. Length-dependent activation in three striated muscle types of the rat. J Physiol. 2002;544:225-36.

23. Lee EJ, Nedrud J, Schemmel P, Gotthardt M, Irving TC and Granzier HL. Calcium sensitivity and myofilament lattice structure in titin N2B KO mice. Arch Biochem Biophys. 2013;535:76-83.

24. Fukuda N, Wu Y, Farman G, Irving TC and Granzier H. Titin isoform variance and length dependence of activation in skinned bovine cardiac muscle. J Physiol. 2003;553:147-54.

25. Konhilas JP, Irving TC and de Tombe PP. Myofilament calcium sensitivity in skinned rat cardiac trabeculae: role of interfilament spacing. Circ Res. 2002;90:59-65.

26. Cazorla O, Wu Y, Irving TC and Granzier H. Titin-based modulation of calcium sensitivity of active tension in mouse skinned cardiac myocytes. Circ Res. 2001;88:1028-35.

27. Farman GP, Walker JS, de Tombe PP and Irving TC. Impact of osmotic compression on sarcomere structure and myofilament calcium sensitivity of isolated rat myocardium. Am J Physiol Heart Circ Physiol. 2006;291:H1847-55.

28. Farman GP, Gore D, Allen E, Schoenfelt K, Irving TC and de Tombe PP. Myosin head orientation: a structural determinant for the Frank-Starling relationship. Am J Physiol Heart Circ Physiol. 2011;300:H2155-60.

29. Ait-Mou Y, Hsu K, Farman GP, Kumar M, Greaser ML, Irving TC and de Tombe PP. Titin strain contributes to the Frank-Starling law of the heart by structural rearrangements of both thin- and thickfilament proteins. Proc Natl Acad Sci U S A. 2016;113:2306-11.

30. Van Der Toorn A, Barenbrug P, Snoep G, Van Der Veen FH, Delhaas T, Prinzen FW, Maessen J and Arts T. Transmural gradients of cardiac myofiber shortening in aortic valve stenosis patients using MRI tagging. Am J Physiol Heart Circ Physiol. 2002;283:H1609-15.

31. Derumeaux G, Mulder P, Richard V, Chagraoui A, Nafeh C, Bauer F, Henry JP and Thuillez C. Tissue Doppler imaging differentiates physiological from pathological pressure-overload left ventricular hypertrophy in rats. Circulation. 2002;105:1602-8.

32. Dosch JC, Szwerc MF, Lin JC, Magovern JA and Edwards JG. Pressure overload induces heterologous expression of the atrial natriuretic factor (ANF) gene. IUBMB Life. 2001;52:315-9.

33. Prestle J, Dieterich S, Preuss M, Bieligk U and Hasenfuss G. Heterogeneous transmural gene expression of calcium-handling proteins and natriuretic peptides in the failing human heart. Cardiovasc Res. 1999;43:323-31.

34. Spragg DD, Leclercq C, Loghmani M, Faris OP, Tunin RS, DiSilvestre D, McVeigh ER, Tomaselli GF and Kass DA. Regional alterations in protein expression in the dyssynchronous failing heart. Circulation. 2003;108:929-32.

35. Kindo M, Gerelli S, Bouitbir J, Hoang Minh T, Charles AL, Mazzucotelli JP, Zoll J, Piquard F and Geny B. Left Ventricular Transmural Gradient in Mitochondrial Respiration Is Associated with Increased Sub-Endocardium Nitric Oxide and Reactive Oxygen Species Productions. Front Physiol. 2016;7:331. 36. MacDonald JR, Oellermann M, Rynbeck S, Chang G, Ruggiero K, Cooper GJ and Hickey AJ. Transmural differences in respiratory capacity across the rat left ventricle in health, aging, and streptozotocin-induced diabetes mellitus: evidence that mitochondrial dysfunction begins in the subepicardium. Am J Physiol Cell Physiol. 2011;300:C246-55. 
37. Marston SB and de Tombe PP. Troponin phosphorylation and myofilament Ca2+-sensitivity in heart failure: increased or decreased? J Mol Cell Cardiol. 2008;45:603-7.

38. Hamdani N, Kooij V, van Dijk S, Merkus D, Paulus WJ, Remedios CD, Duncker DJ, Stienen GJ and van der Velden J. Sarcomeric dysfunction in heart failure. Cardiovasc Res. 2008;77:649-58.

39. Jweied EE, McKinney RD, Walker LA, Brodsky I, Geha AS, Massad MG, Buttrick PM and de Tombe PP. Depressed cardiac myofilament function in human diabetes mellitus. Am J Physiol Heart Circ Physiol. 2005;289:H2478-83.

40. van der Velden J, Papp Z, Zaremba R, Boontje NM, de Jong JW, Owen VJ, Burton PB, Goldmann $P$, Jaquet $K$ and Stienen GJ. Increased Ca2+-sensitivity of the contractile apparatus in end-stage human heart failure results from altered phosphorylation of contractile proteins. Cardiovasc Res. 2003;57:3747.

41. van Der Velden J, Klein L, Zaremba R, Boontje NM, Huybregts MA, Stooker W, Eijsman L, de Jong JW, Visser CA, Visser FC and Stienen GJ. Effects of calcium, inorganic phosphate, and pH on isometric force in single skinned cardiomyocytes from donor and failing human hearts. Circulation. 2001;104:1140-6.

42. Hamdani N, Bishu KG, von Frieling-Salewsky M, Redfield MM and Linke WA. Deranged myofilament phosphorylation and function in experimental heart failure with preserved ejection fraction. Cardiovasc Res. 2013;97:464-71.

43. Konhilas JP, Irving TC, Wolska BM, Jweied EE, Martin AF, Solaro RJ and de Tombe PP. Troponin I in the murine myocardium: influence on length-dependent activation and interfilament spacing. $J$ Physiol. 2003;547:951-61.

44. Cazorla O, Szilagyi S, Vignier N, Salazar G, Kramer E, Vassort G, Carrier L and Lacampagne A. Length and protein kinase $A$ modulations of myocytes in cardiac myosin binding protein C-deficient mice. Cardiovasc Res. 2006;69:370-80.

45. Colson BA, Bekyarova T, Fitzsimons DP, Irving TC and Moss RL. Radial displacement of myosin cross-bridges in mouse myocardium due to ablation of myosin binding protein-C. $\mathrm{J} \mathrm{Mol} \mathrm{Biol.}$

2007;367:36-41.

46. Mamidi R, Gresham KS and Stelzer JE. Length-dependent changes in contractile dynamics are blunted due to cardiac myosin binding protein-C ablation. Front Physiol. 2014;5:461.

47. Colson BA, Bekyarova T, Locher MR, Fitzsimons DP, Irving TC and Moss RL. Protein kinase Amediated phosphorylation of CMyBP-C increases proximity of myosin heads to actin in resting myocardium. Circ Res. 2008;103:244-51.

48. Brenner B and Eisenberg E. Rate of force generation in muscle: correlation with actomyosin ATPase activity in solution. Proc Natl Acad Sci U S A. 1986;83:3542-6.

49. Pan BS and Solaro RJ. Calcium-binding properties of troponin C in detergent-skinned heart muscle fibers. J Biol Chem. 1987;262:7839-49.

50. Hannon JD, Martyn DA and Gordon AM. Effects of cycling and rigor crossbridges on the conformation of cardiac troponin C. Circ Res. 1992;71:984-91.

51. Moss RL, Razumova M and Fitzsimons DP. Myosin crossbridge activation of cardiac thin filaments: implications for myocardial function in health and disease. Circ Res. 2004;94:1290-300.

52. Hofmann PA and Fuchs F. Effect of length and cross-bridge attachment on $\mathrm{Ca} 2+$ binding to cardiac troponin C. Am J Physiol. 1987;253:C90-6.

53. Tanner BC, Farman GP, Irving TC, Maughan DW, Palmer BM and Miller MS. Thick-to-thin filament surface distance modulates cross-bridge kinetics in Drosophila flight muscle. Biophys J. 2012;103:1275-84.

54. Kampourakis T, Sun YB and Irving M. Myosin light chain phosphorylation enhances contraction of heart muscle via structural changes in both thick and thin filaments. Proc Natl Acad Sci U SA. 2016;113:E3039-47. 
55. Kerrick WG, Kazmierczak K, Xu Y, Wang Y and Szczesna-Cordary D. Malignant familial hypertrophic cardiomyopathy D166V mutation in the ventricular myosin regulatory light chain causes profound effects in skinned and intact papillary muscle fibers from transgenic mice. FASEB J. 2009;23:855-65.

56. Yuan CC, Muthu P, Kazmierczak K, Liang J, Huang W, Irving TC, Kanashiro-Takeuchi RM, Hare JM and Szczesna-Cordary D. Constitutive phosphorylation of cardiac myosin regulatory light chain prevents development of hypertrophic cardiomyopathy in mice. Proc Natl Acad Sci U S A. 2015;112:E4138-46. 57. Donal E, Bergerot C, Thibault H, Ernande L, Loufoua J, Augeul L, Ovize M and Derumeaux G. Influence of afterload on left ventricular radial and longitudinal systolic functions: a two-dimensional strain imaging study. Eur J Echocardiogr. 2009;10:914-21.

58. Chetboul V, Escriou C, Tessier D, Richard V, Pouchelon JL, Thibault H, Lallemand F, Thuillez C, Blot $S$ and Derumeaux G. Tissue Doppler imaging detects early asymptomatic myocardial abnormalities in a dog model of Duchenne's cardiomyopathy. Eur Heart J. 2004;25:1934-9.

59. Ait Mou Y, Toth A, Cassan C, Czuriga D, de Tombe PP, Papp Z, Lacampagne A and Cazorla O. Beneficial effects of SR33805 in failing myocardium. Cardiovasc Res. 2011;91:412-9. 